Revista de
Economìd
Contemporâned

\title{
O COMPORTAMENTO DE JOVENS E ADULTOS NO MERCADO DE TRABALHO BRASILEIRO COM RELAÇÃO À PERMANÊNCIA NO EMPREGO
}

\author{
Raquel Pereira Pontes ${ }^{a}$ \\ Marcio Nora Barbosa ${ }^{b}$ \\ Leonel dos Santos Silveira ${ }^{c}$ \\ Lorena Vieira Costa ${ }^{d}$
}

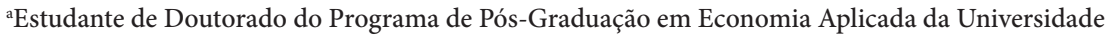
Federal de Viçosa (UFV).

bEstudante de Doutorado do Programa de Pós-Graduação em Economia Internacional da Universidade do Vale do Rio dos Sinos (UNISINOS).

'Professor substituto do Instituto de Ciências Econômicas, Administrativas e Contábeis da Universidade Federal do Rio Grande (FURG).

${ }^{\text {d} P r o f e s s o r a ~ A d j u n t a ~ d o ~ D e p a r t a m e n t o ~ d e ~ E c o n o m i a ~ R u r a l ~ d a ~ U F V . ~}$
\end{abstract}

Artigo recebido em 24/03/2018 e aceito para publicação em 16/08/2018.

RESUMO: A permanência do trabalhador no mercado de trabalho é de grande importância para a economia do país. Isso se dá, principalmente, devido ao ganho de capital humano adquirido pelo trabalhador, especialmente quando jovem. Dentro dessa perspectiva, este trabalho busca analisar as incidências de rotatividade entre empregos dos jovens brasileiros nos anos de 2001, 2009 e 2015, bem como avaliar os aspectos que determinam maiores níveis de rotatividade, especificamente, para o ano de 2015. Para tanto, com base nos dados da Pesquisa Nacional por Amostra de Domicílios (PNAD) do ano de 2015, aplicou-se o modelo econométrico Logit Ordenado para identificar os fatores que se associam ao comportamento de indivíduos em diferentes faixas etárias, no que concerne às suas propensões a estarem em um nível de alta ou baixa rotatividade no

Correspondência para: Raquel Pereira Pontes

Contato: raquel_sjn@hotmail.com 
mercado de trabalho. Os resultados evidenciaram que pessoas de 18 a 35 anos são mais propensas a saírem do emprego e que fatores como maior escolaridade diminui a probabilidade de ter alto nível de rotatividade no mercado de trabalho.

PALAVRAS-CHAVE: rotatividade; jovens; mercado de trabalho; Logit Ordenado.

CLASSIFICAÇÃO JEL: J01; J63. 


\title{
THE BEHAVIOR OF YOUNG PEOPLE AND ADULTS IN THE BRAZILIAN LABOR MARKET IN RELATION TO THEIR PERMANENCE IN EMPLOYMENT
}

\begin{abstract}
The permanence of workers in the labor market is of great importance for the economy of acountry. This is mainly due to gains in human capital for workers, especially when young. From the perspective of young workers, this study seeks to analyze the incidence of labor turnover among Brazilian youth in the years 2001, 2009 and 2015, as well as to determine the causes behind highest turnover levels specifically for the year 2015. Based on data from the National Sample Survey (PNAD) of 2015, the logit ordered econometric level has been applied to identify factors that are associated with the behavior of individuals in different age groups, with respect to their propensities to be at a high or low turnover level in the labor market. The results show that people between the ages of 18 and 35 are more likely to leave their jobs and that factors such as higher schooling reduce the likelihood of high turnover in the labor market.
\end{abstract}

KEYWORDS: labor turnover; young people; job market; Logit Ordered. 


\section{INTRODUÇÃO}

A permanência do trabalhador no emprego é de suma importância para o mercado de trabalho, devido à experiência que se é obtida ao longo da vida desse indivíduo, o que resulta em uma melhora na sua produtividade. As organizações econômicas necessitam não somente explorar habilidades e educação dos trabalhadores, mas também mantê-los leais e capazes. A questão central desta ideia baseia-se, principalmente, no argumento da eficiência econômica do capital humano, conforme analisado por Becker (1993).

Além disso, uma grande rotatividade no mercado de trabalho gera pressões sobre os gastos sociais governamentais, particularmente com programas como seguro desemprego. De fato, Monte e Penido (2008) ressaltam que uma maior permanência no emprego tende a diminuir o custo social. ${ }^{1}$ Para as empresas, a rotatividade de empregados envolve custos relacionados com o treinamento e com a baixa produtividade inicial a cada novo funcionário. Apesar disso, tem-se observado uma grande rotatividade da força de trabalho no Brasil no período analisado neste estudo, ou seja, de 2001 a 2015, principalmente entre trabalhadores jovens e jovens em idade adulta.

Além da preocupação em manter o trabalhador por mais tempo em seu emprego, de acordo com Ramos (2007), outros fatores, como mudanças na economia brasileira, também fizeram com que os demandantes por trabalho exigissem maiores qualificações, $o$ que por sua vez acabou por influenciar na decisão dos jovens em retardar a oferta de mão de obra. Camarano et al. (2003) também argumentam que as mudanças sociais constatadas à época deste estudo refletiram na opção dos jovens pelo adiamento da saída da casa dos pais, devido à instabilidade no mercado de trabalho e à opção entre esses indivíduos, por uma formação mais extensa, que demanda mais tempo na escola. Além disso, a recente instabilidade econômica na qual o país encontra-se, perceptível a partir do ano de 2014, pode impactar sobre a rotatividade do mercado de trabalho. Todos esses movimentos, observados a partir dos anos 2001, exercem grande influência sobre diferentes características do mercado de trabalho brasileiro e sobre o comportamento dos jovens, afetando a rotatividade entre empregos.

Para a avaliação da dimensão desse movimento, torna-se fundamental a construção do perfil dos indivíduos que possuem maiores chances de estar em um nível mais alto de rotatividade no emprego. Essa análise possibilita a indicação de grupos de pessoas com características que as tornam mais prováveis de estar em situação de alta rotatividade no mercado de trabalho e, por sua vez, subsidiar ações públicas e privadas que objetivem contornar essa tendência.

\footnotetext{
1 O custo social corresponde aos custos privados e aos custos percebidos pela sociedade.
} 
Diante do fato de que existem níveis de rotatividade diferentes, é possível que haja diferenças entre os fatores que afetam cada um deles, fato ainda não estudado com rigor pela literatura recente. Assim, neste estudo avaliam-se os diversos níveis de rotatividade no mercado de trabalho pelos jovens brasileiros, considerando que se trata de um resultado de escolha ordenada. Este trabalho objetiva, então, analisar o comportamento de jovens e adultos (separados por faixas de idades) no mercado de trabalho brasileiro em relação à rotatividade desses indivíduos no emprego nos anos de 2001, 2009 e 2015, utilizando dados da PNAD. Além disso, visa a identificar, especificamente para o ano de 2015, por meio de um modelo econométrico Logit Ordenado, os fatores que predizem as chances de estes sujeitos estarem em um nível mais alto ou mais baixo de impermanência no emprego, além de verificar outros importantes aspectos que podem definir o grau de rotatividade desses jovens, como a educação e o gênero.

Este artigo está estruturado em cinco seções. Na primeira delas, nesta introdução, é apresentada a importância a respeito do estudo sobre rotatividade e permanência do trabalhador no emprego. Na segunda, apresenta-se uma revisão da teoria e de estudos empíricos sobre a rotatividade no mercado de trabalho. A terceira seção apresenta a metodologia e descreve a fonte de dados, as variáveis e o método utilizados. Os resultados da análise e as suas discussões são trazidos na seção quatro. Na última seção, são apresentadas as considerações finais.

\section{ROTATIVIDADE NO MERCADO DE TRABALHO: UMA REVISÃO TEÓRICA E ANALÍTICA DOS ESTUDOS EMPÍRICOS}

Na literatura encontra-se a necessidade de analisar o desemprego, bem como a mobilidade e a rotatividade do emprego sob a ótica da Teoria do Capital Humano e da Teoria da Procura. Estudos de Becker (1975), Oi (1961) e Parsons (1972) elucidaram a conexão entre investimentos específicos na firma e rotatividade no trabalho. Outra teoria de interesse vem dos modelos estocásticos da mobilidade do trabalho, ou seja, o modelo mover-stayer de autores como Blumen, Kogan e McCarthy(1955) e Singer e Spilerman (1976) (MINCER, 1993).

A teoria mover-stayer tem uma visão latente segundo a qual a necessidade de mobilidade da pessoa pode não acontecer somente no emprego, mas em outras dimensões da sua vida, sendo assim da própria natureza humana e, devido a esta instabilidade, os indivíduos podem ser menos produtivos. Dessa maneira, a teoria divide os indivíduos em dois tipos: os de alta produtividade (stayers) e os de baixa produtividade (movers), relacionando características pessoais não observadas com as atitudes observadas que demonstram chances de impermanência no emprego. 
$\mathrm{Na}$ Teoria do Capital Humano, desenvolvida por Gary Becker, tem-se a ideia de que as empresas enfrentam um problema de como garantir a continuidade dos trabalhadores no emprego, visto que elas têm um gasto significativo com investimentos nesses empregados. Isso porque, se o funcionário sai da firma, a empresa tem perda de capital humano investido e maior custo ao ter que capacitar um novo empregado. Esse treinamento é subdividido por Becker (1962) em: treinamento geral, que aumenta a produtividade do trabalhador independentemente de em que empresa esteja empregado; e treinamento específico, que aumenta a produtividade do trabalhador, mas somente para a empresa na qual esteja empregado. A solução vista por Becker seria a divisão dos custos do treinamento entre a empresa e o trabalhador, em que ambos manteriam o relacionamento de trabalho.

Outro importante modelo abordando o problema da permanência/impermanência é aquele proposto pela Teoria da Procura por Trabalho (Job Search Theory), em que o indivíduo determina um salário reserva (remuneração limiar) antes de receber a proposta, e assim toma a sua decisão de aceitar ou não o emprego com base nesse salário reserva. Segundo essa teoria, apenas desempregados buscam por empregos.

Burdett (1978) aprimora a Teoria da Procura por Trabalho com o modelo On the Job Search, estabelecendo que não somente desempregados buscam emprego, mas também pessoas com vínculo empregatício. Essa afirmação é feita com base em uma situação específica: quando o indivíduo desempregado tem dois salários de reserva, se um deles é satisfatório ele aceita, mas continua procurando emprego para alcançar o maior salário de reserva, e a pessoa empregada vai aceitar nova oferta se esta for maior que sua remuneração atual. Assim, a saída do trabalhador do emprego pode estar associada a uma oferta de salário maior.

Burdett (1978) também conclui que a idade do trabalhador e o fato de ele estar empregado têm relação negativa com a saída do emprego por envolver também tempo de participação da força de trabalho. Barron e McCafferty (1977), em um modelo similar ao de Burdett (1978), observam que o total de saídas dos empregos aumenta com o crescimento da demanda agregada do trabalho.

O efeito da idade do trabalhador na rotatividade do mercado trabalho também está relacionado com a sua satisfação no emprego. Como constatado por Sparrow (1996), um novo mercado com fatores, como a desregulamentação, regras implícitas no contrato e novas tecnologias, pode estar afetando a decisão de trocar de emprego.

Outra teoria interessante para explicar a rotatividade no mercado é a teoria do Job Shopping (JOHNSON, 1978) baseada na informação incerta e imperfeita. A teoria explica que ao longo do tempo os trabalhadores e as empresas obtêm informações sobre o posto de trabalho e sobre o funcionário, respectivamente, de maneira que somente quando ocorre a compatibilidade entre os interesses de ambos a relação de trabalho sobrevive. 


\subsection{ESTUDOS EMPÍRICOS NO BRASIL}

No Brasil, conforme Monte e Penido (2008), podem-se identificar três linhas de estudos sobre a rotatividade no mercado de trabalho: os que buscam mensurar a rotatividade, como Corseuil, Ribeiro e Santos (2003); aqueles que procuram avaliar a relação entre a alta rotatividade e o baixo custo da demissão no Brasil, além de avaliar também as políticas públicas que acabam incentivando a alta rotatividade, como Barros, Corseuil e Foguel (2000); e, por último, aqueles que buscam analisar os determinantes da rotatividade do emprego, como os trabalhos de Menezes Filho e Pichetti (2000), de Orellano e Pichetti (2001) e de Monte e Penido (2008).

Menezes-Filho e Pichetti (2000) averiguaram os determinantes da duração esperada no emprego na região metropolitana de São Paulo para o ano de 1997- com dados da Pesquisa Mensal de Emprego (PME) - e constataram que chefes de família e pessoas que já haviam trabalhado antes têm maior duração média no emprego.

Orellano e Pichetti (2001), com dados de uma pesquisa da região metropolitana de São Paulo (a Pesquisa de Emprego e Desemprego - PED), no período de 1985 a 1999, buscaram identificar quais eram os determinantes das saídas e das demissões no emprego. Os principais fatores observados pelos autores são: o gênero, visto que as mulheres são mais propensas a abandonar o emprego; a idade, evidenciando uma maior rotatividade por parte dos jovens; e, por fim, o fato de o indivíduo estar na condição de chefe de família, visto que esse grupo apresentou menores chances de saída do emprego.

Corseuil et al. (2013) investigaram a rotatividade dos jovens no mercado de trabalho formal brasileiro, por meio da análise dos fluxos de entrada e de saída dos trabalhadores, com base nos dados da Relação Anual de Informações Sociais (Rais), do Ministério do Trabalho e Emprego (MTE), para o período de 1996 a 2010. Entre os seus resultados, os autores verificaram que os jovens apresentam alta rotatividade no mercado de trabalho e concluíram que isto pode dificultar o aumento da produtividade futura e de salários, por baixo acúmulo de capital humano.

Monte e Penido (2008) analisaram os determinantes da duração esperada no emprego, especificando a área rural e urbana para a região nordeste do Brasil para os anos de 1993 e 2003, utilizando dados da PNAD. Segundo os autores, a duração no emprego é maior para homens, chefes de família, brancos e trabalhadores com idade mais elevada.

Gonzaga, Maloney e Mizala (2003) analisaram a rotatividade no Brasil com referência nas leis trabalhistas no país. Os resultados demonstraram que o custo de rescisão do trabalho aumentou depois da constituição de 1988 - esse custo se constituiu em uma multa de $40 \%$ do Fundo de Garantia do Tempo de Serviço (FGTS) a ser paga pelo empregador para o trabalhador demitido sem justa causa (antes da constituição de 1988 a multa era de $10 \%$ do FGTS). Outro fator de influência para esse aumento foi a implementação da lei complementar no 110 de 29 de junho de 2001, que passou a exigir que o empregador pagasse também 10\% do FGTS para o governo pela demissão 
sem justa causa. Os autores concluíram que, com esse aumento de custo, a rotatividade dos trabalhadores tenderia a diminuir.

Em se tratando especificamente de jovens e adultos no mercado de trabalho brasileiro, Albuquerque e Pero (2009) analisaram a mobilidade de emprego entre membros desse público por meio de dados em painel, com base em informações do banco de dados do Ministério do Trabalho e Emprego (MTE), para o período de 1996 a 2005. Entre seus resultados, os autores perceberam uma relação positiva entre a rotatividade e os salários, mas de forma decrescente, ou seja, a partir dos cinco anos do primeiro emprego a rotatividade gera ganhos salariais menores.

Diante da literatura existente, este trabalho traz uma contribuição ao propor uma análise mais recente, e para todo o Brasil, da situação dos jovens quanto à rotatividade no mercado de trabalho. Além disso, este estudo reconhece o fato de que há diferentes níveis de rotatividade e, assim, a estratégia empírica deve considerar este fato.

\section{METODOLOGIA}

\subsection{FONTE DE DADOS E DEFINIÇÃO DAS VARIÁVEIS}

Neste trabalho, busca-se verificar o papel de diferentes características dos indivíduos sobre diferentes níveis de impermanência no mercado de trabalho brasileiro. Utilizou-se um modelo econométrico para se observar a chance de os jovens estarem em alta ou baixa rotatividade dentro do mercado de trabalho, segundo suas características pessoais, educacionais, familiares, trabalhistas e geográficas. Para tanto, utilizou-se do método Logit Ordenado, conforme explicado a seguir.

Os dados foram extraídos da Pesquisa Nacional por Amostra de Domicílios (PNAD) do ano de 2015 (IBGE, 2017). A PNAD 2015 contém uma amostra de 356.904 pessoas, que correspondem a 204.860.101 pessoas com a ponderação da pesquisa. A PNAD utiliza de um plano amostral estratificado, conglomerado que, dependendo do estrato, pode ser em dois ou três estágios (IBGE, 2016).

A PNAD possui dados referentes à permanência do trabalhador no emprego, extraídos de perguntas como "De quantos trabalhos saiu no período de captação de 358 dias" e "Saiu de algum trabalho no período de captação de 358 dias", dirigindo-se a pessoas que estavam no trabalho na semana de referência ou que não estavam trabalhando, mas estavam buscando trabalho².

2 Este trabalho preocupou-se em analisar trabalhadores que estavam em serviço na semana de referência ou não estavam ocupados na semana de referência, mas estavam procurando emprego, pois o interesse nesta análise é 
Assim, determinou-se a variável dependente desta análise: a rotatividade. Ela foi categorizada de três modos distintos: alta, se o indivíduo saiu de dois ou mais empregos no período de 358 dias; baixa, se saiu somente de um posto; e nula, se o indivíduo não saiu do emprego, conforme mostra a Figura 1:

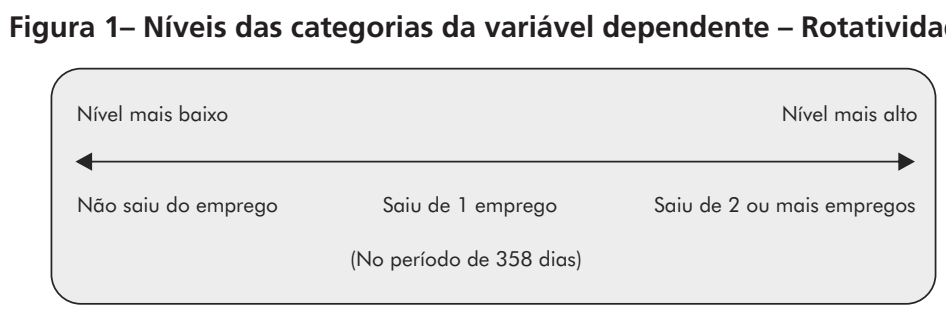

Para a definição da amostra, utilizaram-se dados de pessoas com 14 anos ou mais, desde que estivessem trabalhando na semana de referência ou desempregadas em busca de emprego. As variáveis de interesse analisadas foram os intervalos de idade e, de forma similar ao estudo de Andrade (2008), a divisão foi feita da seguinte forma, a fim de facilitar a leitura: os jovens de 14 a 17 anos ou "Jovens-Aprendizes" ou "Jovens-Adolescentes" definidos como Jovens1; os de 18 a 24 anos ou "Jovens-Jovens" como Jovens2; os de 25 a 35 anos ou "Adultos-Jovens" como Adultos1; os de 36 a 50 anos ou "Adultos", definido como Adultos2; e os maiores de 50 anos ou “Adultos-Idosos”, como Adultos3.

Ramos e Carneiro (2002) observam que a rotatividade pode estar associada ao setor de atividade e região demográfica. Desta maneira, é relevante considerar as regiões demográficas e censitárias do Brasil como variáveis explicativas

Ademais, inserem-se como variáveis explicativas características do indivíduo, como raça, sexo, nível de escolaridade, se está estudando, se é chefe de família ou mãe com filhos, quantidade de pessoas no domicílio, renda domiciliar per capita e se é associado a algum sindicato.

\subsection{MODELO LOGIT ORDENADO}

O modelo Logit Ordenado faz parte de modelos multinomiais, em que a variável dependente é tratada como um reflexo de categorias ordenadas. Conforme Cameron e Trivedi (2005), os modelos gerais multinomiais tratam de casos em que a variável de-

o de avaliar a rotatividade. Assim, é importante que a pessoa volte à atividade, pois caso não esteja trabalhando ou procurando emprego, não há a possibilidade de saber se este voltará para o mercado de trabalho. 
pendente assume diferentes valores discretos, representando diferentes alternativas possíveis. Tem-se $m$ alternativas, em que a variável dependente $Y$ assume o valor $j$ quando a $j$-ésima alternativa é escolhida. No caso do Logit Ordenado, há uma ordenação natural quanto a essas categorias.

Segundo Long e Freese (2001) e Cameron e Trivedi (2005), o modelo Logit Ordenado possui uma variável latente que varia de $-\infty$ a $\infty$.

Tal variável, segundo Cameron e Trivedi (2005), não é completamente observada. Nos modelos de resposta binária, essa racionalidade pode ser útil para fornecer uma abordagem mais teórica ao problema. Neste trabalho, pode-se pensar em uma variável latente, na medida em que ela atinge diferentes limiares, indicativa da insatisfação ou da maior propensão ao risco de demissão do trabalhador (em detrimento da estabilidade no trabalho), fatores que determinam o número de vezes que o indivíduo optará por sair de seu emprego. Assim, a variável latente pode ser importante por ser um índice de uma propensão não observada de que um evento de interesse ocorra ou, em outros casos, por se apresentar como a diferença na utilidade, caso o evento ocorra (CAMERON e TRIVEDI, 2005).

Desta forma, um modelo Logit Ordenado com base em um modelo de índice com variável latente pode ser especificado como:

$$
Y_{i}^{*}=X_{i}^{\prime} B+u_{i}
$$

Em (1) $Y_{i}^{*}$ representa a variável latente do indivíduo $i, X_{i}$ as variáveis explicativas, $\beta$ os parâmetros e $u_{i}$ o ruído aleatório. Deste modo, conforme Cameron e Trivedi (2005), a variável latente $Y_{i}^{*}$ atravessa uma série de limites (thresholds) desconhecidos, fazendo com que o indivíduo se mova entre as alternativas.

$$
Y_{i}=j \text { se } \alpha_{j-1}<Y_{i}^{*} \leq \alpha_{j}
$$

Nesse modelo apresentado, $\alpha_{1}, \ldots, \alpha_{m-1}$ são os parâmetros dos limites $(m-1)$ e que $\alpha_{0}=-\infty$ e $\alpha_{m}=\infty$. Então,

$$
\begin{aligned}
P_{r}\left[Y_{i}=j\right] & =P_{r}\left[\alpha_{j-1}<Y_{i}^{*} \leq \alpha_{j}\right] \\
& =P_{r}\left[\alpha_{j-1}<X_{i}^{\prime} B+\mu_{i} \leq \alpha_{j}\right] \\
& =P_{r}\left[\alpha_{j-1}-X_{i}^{\prime} B<\mu_{i} \leq \alpha_{j}-X_{i}^{\prime} B\right] \\
& =F\left(\alpha_{j}-X_{i}^{\prime} B\right)-F\left(\alpha_{j-1}-X_{i}^{\prime} B\right)
\end{aligned}
$$


Em (3), F representa a função densidade acumulada do erro $\mu_{i}$.

No modelo Logit Ordenado, por meio da máxima verossimilhança obtêm-se parâmetros consistentes e eficientes, desde que a função esteja corretamente especificada.

Neste trabalho utilizam-se três categorias:

$$
\begin{cases}Y_{i}=0=\text { nula rotatividade } & \text { se } \alpha_{0}=-\infty \leq Y_{i}^{*}<\alpha_{1} \\ Y_{i}=1=\text { baixa rotatividade } & \text { se } \alpha_{1} \leq Y_{i}^{*}<\alpha_{2} \\ Y_{i}=2=\text { alta rotatividade } & \text { se } \alpha_{2} \leq Y_{i}^{*}<\alpha_{3}=\infty\end{cases}
$$

Em (4) um valor de $Y_{i}$ muito baixo representa a permanência do trabalhador no emprego, $Y_{i}=1$ representa a baixa rotatividade; e $Y_{i}=2$, alta rotatividade no emprego no último ano.

Neste modelo, conforme Cameron e Trivedi (2005), a interpretação pode ser feita analisando-se diretamente os sinais dos coeficientes. Nesse sentido, se o estimador é positivo, tem-se um aumento na chance de se estar em uma categoria mais alta, diminuindo, portanto, a chance de se estar em uma categoria mais baixa. O modelo permite analisar também os efeitos marginais:

$$
\frac{\partial P_{r}\left[y_{i}=j\right]}{\partial X_{i}}=\left\{F^{\prime}\left(\alpha_{j-1}-X_{i}^{\prime} \beta\right)-F^{\prime}\left(\alpha_{j}-X_{i}^{\prime} \beta\right)\right\} \beta
$$

A equação (5) demonstra a probabilidade prevista de uma categoria específica para cada valor de determinada variável. Por exemplo, pode-se obter a probabilidade prevista de se estar em uma situação de nula rotatividade no mercado de trabalho, dado que frequenta ou não a escola. A interpretação dos efeitos marginais é semelhante à do modelo Multinomial Logit Padrão (não ordenado).

O modelo Logit Ordenadotrabalha com a hipótese de que a inclinação do modelo é a mesma - hipótese de chances proporcionais, também chamada de hipótese de regressão paralela. Ou seja, conforme IDRE-UCLA (2016), a relação entre cada par de resultados de grupos é a mesma, indicando, por exemplo, que coeficientes que descrevem a relação entre a menor e todas as maiores categorias são os mesmos que explicam a relação entre a próxima menor categoria e todas as outras mais altas.

Assim, se o modelo aqui estudado não confirmar esta hipótese, pode-se lidar com esta situação das seguintes formas, conforme Williams (2017): ignorar a não confir- 
mação, prática bastante comum conforme o autor; tratar como uma alternativa não ordinal como um Logit Multinomial; considerar alternativa ordinal como o Logit Ordenado Generalizado ou uma abordagem intermediária que é Logit Ordenado - Probabilidades Proporcionais Parciais.

Diante de testes de estabilidade dos coeficientes, o modelo indicado neste traba$l_{\text {ho }}^{3}$ é o Logit Ordenado de Probabilidades Proporcionais Parciais ${ }^{4}$. Nele permite-se que somente os coeficientes que confirmaram a hipótese sejam os mesmos para todo $j$, enquanto os outros coeficientes podem diferir (WILLIAMS, 2017). Assim, relaxa-se a hipótese somente para as variáveis que efetivamente não afirmaram a hipótese.

Devido ao uso do modelo Logit Ordenado de Probabilidades Proporcionais Parciais, em que variáveis que violaram a hipótese de regressão paralela podem diferir entre as categorias ordenadas, os resultados do modelo são interpretados da mesma forma dos modelos de regressão logística binária. No caso do atual modelo, no qual há $m$ categorias, tem-se uma série de regressões logísticas binárias, ou seja: comparações entre a categoria nula rotatividade versus as categorias baixa rotatividade e alta rotatividade; e categoria baixa rotatividade versus categoria alta rotatividade.

\section{RESULTADOS E DISCUSSÕES}

\subsection{COMPORTAMENTO DOS NÍVEIS DE ROTATIVIDADE NO MERCADO DE TRABALHO BRASILEIRO}

No período de 15 anos, de 2001 a 2015, as frequências de trabalhadores entre os níveis de rotatividade ${ }^{5}$ alteraram-se bastante, com uma mudança de comportamento que parece refletir principalmente a situação econômica do país. Como observado na Tabela 1, a quantidade total de trabalhadores ${ }^{6}$ entre os níveis de rotatividade foi mais elevada em 2009, período de maior estabilidade econômica no país, considerando os períodos analisados.

3 Referente à hipótese de chance proporcional, foi realizado o teste Brant, que no caso de rejeitar hipótese nula, torna necessáriaa estimação do Logit Ordenado de Chances Proporcionais Parciais.

4 Para mais informação sobre o modelo e a interpretação deste modelo, ver Williams (2006 e 2016).

5 Nula Rotatividade: se o indivíduo não saiu do emprego; Baixa Rotatividade: se saiu somente de um posto; e Alta Rotatividade: se o indivíduo saiu de dois ou mais empregos no período de 358 dias. Mais informações na seção de metodologia do trabalho.

6 Trabalhadores com 14 anos de idade ou mais, empregados e não empregados, buscando emprego na semana de referência e que saíram do último emprego no período de 358 dias da data da pesquisa. 
Tabela 1 - Número de trabalhadores entre os níveis de rotatividade (captação de 358 dias) no Brasil nos anos de 2001, 2009 e 2015

\begin{tabular}{lcccccc}
\hline \multirow{2}{*}{$\begin{array}{l}\text { Níveis de } \\
\text { rotatividade no } \\
\text { Brasil }\end{array}$} & \multicolumn{2}{c}{2001} & \multicolumn{2}{c}{2009} & \multicolumn{2}{c}{2015} \\
\cline { 2 - 6 } & Freq. & $\%$ & Freq. & $\%$ & Freq. & $\%$ \\
\hline Nula & 118.553 .830 & 93,37 & 185.358 .622 & 95,55 & 159.322 .908 & 96,45 \\
Baixa & 7.616 .966 & 6,00 & 7.864 .580 & 4,05 & 5.518 .177 & 3,34 \\
Alta & 802.101 & 0.63 & 771.921 & 0,40 & 343.173 & 0,21 \\
Total & 126.955 .670 & 100 & 193.995 .123 & 100 & 165.184 .258 & 100 \\
\hline
\end{tabular}

Fonte: Elaboração própria, com basenos dados da PNAD (2001, 2009 e 2015).

Verifica-se que houve um aumento no percentual de trabalhadores que não saíram do emprego no período de 358 dias anteriores à data da entrevista da pesquisa da PNAD entre 2001 e 2015. Do mesmo modo, a baixa ou alta rotatividade foram menores em 2015. Esses dados podem refletir uma resposta dos indivíduos ao período de instabilidade econômica e política do país. À medida que a demanda por mão de obra se reduz, as pessoas tendem a se garantir no emprego.

Outro fator também relevante foi a alteração das regras (meses trabalhados e quantidade de parcelas de seguro desemprego recebidas) para o recebimento do Seguro Desemprego - Lei no 13.134/2015, em que para a solicitação da primeira parcela é necessário ter trabalhado pelo menos 12 meses e não seis meses como em períodos anteriores. Para a segunda solicitação é preciso ter trabalhado pelo menos nove meses e para as demais solicitações (a partir da terceira) o tempo de trabalho mínimo é de seis meses.

Em geral, os dados mostram uma tendência de baixa rotatividade dos trabalhadores brasileiros: mais de $90 \%$ dos trabalhadores estavam em situação de permanência no emprego, em todos os anos. No entanto, deve-se ressaltar que esta análise não leva em conta diferentes níveis de rotatividade entre indivíduos com diferentes características.

\subsection{ANÁLISE DESCRITIVA}

$\mathrm{Na}$ Tabela 2, podem ser observadas as principais estatísticas descritivas das variáveis de interesse deste estudo. Verifica-se que $8,67 \%$ da população-alvo são de Jovens 1 ; 13,85\%, de Jovens $2 ; 21,51 \%$, de Adultos $1 ; 26,29 \%$, de Adultos2; e 29,67\% são de Adultos3. Referentemente ao sexo e à cor ou raça, quase $48 \%$ dos trabalhadores ou desempregados em busca de trabalho são do sexo masculino e aproximadamente $42 \%$ são declarados da cor ou raça branca ou amarela. 
Tabela 2 - Estatísticas descritivas das variáveis utilizadas no modelo

\begin{tabular}{|c|c|c|c|}
\hline Variável & Obs. & Média & Erro Padrão \\
\hline Jovens1 & 286462 & 0,0867 & 0,0005 \\
\hline Jovens2 & 286462 & 0,1385 & 0,0006 \\
\hline Adultos1 & 286462 & 0,2151 & 0,0007 \\
\hline Adultos2 & 286462 & 0,2629 & 0,0008 \\
\hline Adultos3 & 286462 & 0.2967 & 0,0008 \\
\hline Masculino & 286462 & 0,4783 & 0,0009 \\
\hline Cor/Raça: Branca e Amarela & 286462 & 0,4248 & 0,0009 \\
\hline Escolaridade & 286462 & 9,20 & 0,0083 \\
\hline Frequenta escola & 286462 & 0,1512 & 0,0007 \\
\hline Renda domiciliar per capita & 286462 & 1138,74 & $2.73 e+08$ \\
\hline Número de componentes & 286462 & 3,5875 & 0,0031 \\
\hline Chefe de família & 286462 & 0,4318 & 0,0009 \\
\hline Mãe com filhos & 286462 & 0,0205 & 0,0002 \\
\hline Associados em sindicato & 179144 & 0,1856 & 0,0009 \\
\hline Urbana & 286462 & 0,8591 & 0,0006 \\
\hline Norte & 286462 & 0,1514 & 0,0006 \\
\hline Nordeste & 286462 & 0,2837 & 0,0008 \\
\hline Sul & 286462 & 0,1574 & 0,0007 \\
\hline Sudeste & 286462 & 0,3037 & 0,0008 \\
\hline Centro-Oeste & 286462 & 0,1037 & 0,0006 \\
\hline
\end{tabular}

Fonte: Elaboração própria com base nos resultados da análise.

Quanto à educação, a escolaridade média é de 9,2 anos de estudo, o que corresponde ao ensino médio incompleto; e 15\% dos trabalhadores ou desempregados em busca de emprego estavam frequentando a escola na semana de referência.

Em média, os entrevistados residem em domicílio cuja composição familiar varia de três a quatro pessoas. Cerca de $43 \%$ das pessoas analisadas são chefes de família e $2 \%$ são mães com filhos.

Ao analisar o local de residência dos indivíduos da amostra, foi verificado que aproximadamente $15 \%$ das pessoas analisadas residiam no norte do país; $28 \%$, na região Nordeste; $16 \%$, no Sul; 30\%, no Sudeste; e 10\%, no Centro-Oeste. Em se tratando das regiões censitárias, $86 \%$ da população analisada residia em área urbana. Referentemente às relações trabalhistas, evidenciou-se que $18,56 \%$ da amostra é associada a algum tipo de sindicato. 
A Tabela 3 apresenta uma análise descritiva dos níveis de rotatividade do trabalhador no mercado de trabalho, assim como a relação desses níveis com as faixas etárias dos empregados, expondo a distribuição desses níveis por intervalos de idade, para o ano base de 2015 .

Tabela 3 - Níveis de rotatividade por intervalos de idade para o ano de 2015

\begin{tabular}{|c|c|c|c|c|c|}
\hline \multirow[b]{2}{*}{$\begin{array}{l}\text { Níveis de } \\
\text { Rotatividade }\end{array}$} & \multicolumn{5}{|c|}{ Intervalos de Idades } \\
\hline & $\begin{array}{c}\text { Jovens } 1 \\
\text { (14 a } 18 \text { anos) } \\
(\%)\end{array}$ & $\begin{array}{c}\text { Jovens2(18 a } 24 \\
\text { anos) (\%) }\end{array}$ & $\begin{array}{c}\text { Adultos1 }(25 \text { a } \\
35 \text { anos })(\%)\end{array}$ & $\begin{array}{c}\text { Adultos } 2 \\
\text { (36 a } 50 \text { anos) } \\
(\%)\end{array}$ & $\begin{array}{c}\text { Adultos3 (>50 } \\
\text { anos) } \\
(\%)\end{array}$ \\
\hline Nula & 99,18 & 93,35 & 93,66 & 96,42 & 99,05 \\
\hline Baixa & 0,77 & 6,25 & 5,99 & 3,38 & 0,88 \\
\hline Alta & 0,06 & 0,39 & 0,35 & 0,20 & 0,07 \\
\hline Total & 100,00 & 100,00 & 100,00 & 100,00 & 100,00 \\
\hline
\end{tabular}

Fonte: Elaboração própria com base nos resultados da análise.

Considerando o total de pessoas de cada faixa etária, foi constatado que $99,18 \%$ dos Jovens 1 estiveram em um nível de nula rotatividade no período analisado, assim como 96\% dos Adultos2 e 99\% das pessoas com 50 ou mais anos de idade. Evidenciou-se, assim, que a baixa rotatividade é mais observada entre os Jovens2 (6,25\%), seguidos pelos Adultos1 (5,99\%) e pelos Adultos2 (3,38\%). A alta rotatividade possui maior intensidade entre os Jovens $2(0,39 \%)$ e baixa frequência entre as pessoas com 50 ou mais anos de idade $(0,07 \%)$.

Essas informações evidenciam a existência de uma diferenciação expressiva entre jovens, adultos e idosos na questão da rotatividade no mercado de trabalho. Tais aspectos, como maior alternância por parte dos Jovens2 e dos Adultos1, repercutem no capital humano existente no Brasil e, consequentemente, na produtividade desses trabalhadores, bem como na percepção de maiores custos sociais pelas empresas no país.

\subsection{PERFIL DOS TRABALHADORES E NÍVEIS DE ROTATIVIDADE NO BRASIL}

Com o propósito de averiguar o perfil dos trabalhadores em termos de seus níveis de rotatividade, considerando principalmente a faixa etária dos mesmos, estimou-se por meio do Logit Ordenado a razão de chance de o indivíduo possuir maior ou menor rotatividade no mercado de trabalho. 
Foram realizados testes para verificar as medidas de ajuste, os quais demonstraram que o modelo possui bom ajustamento. Em se tratando da hipótese de chance proporcional, o teste Brant rejeitou essa hipótese, tornando necessária a estimação do Logit Ordenado de Chances Proporcionais Parciais.

Apresenta-se, a seguir, os resultados do modelo nas Tabelas 4 e 5. Na Tabela 4, têm-se os efeitos de diferentes intervalos de idades dos indivíduos sobre as chances de maior alternância. Tabela 4 - Efeitos dos intervalos de idade sobre os níveis de
rotatividade dos trabalhadores brasileiros, 2015

\begin{tabular}{|c|c|c|c|c|}
\hline \multirow{3}{*}{ Variáveis } & \multicolumn{4}{|c|}{ Nível de Rotatividade } \\
\hline & \multicolumn{2}{|c|}{ Nula } & \multicolumn{2}{|c|}{ Baixa } \\
\hline & \multicolumn{2}{|c|}{ Coeficiente Razão de Chance } & \multicolumn{2}{|c|}{ Coeficiente Razão de Chance } \\
\hline \multicolumn{5}{|l|}{ Intervalo de idade } \\
\hline Jovens1 & $\begin{array}{c}-0,1484 \\
(0,0937)\end{array}$ & 0,8621 & $\begin{array}{l}-0,1484 \\
(0,0937)\end{array}$ & 0,8621 \\
\hline Jovens2 & $\begin{array}{c}2,0001^{* * *} \\
(0,0500)\end{array}$ & 7,3947 & $\begin{array}{c}1,6895^{* * *} \\
(0,1444)\end{array}$ & 5,4169 \\
\hline Adultos 1 & $\begin{array}{c}1,9509^{* * *} \\
(0,0455)\end{array}$ & 7,0352 & $\begin{array}{c}1,5788^{\star * *} \\
(0,1591)\end{array}$ & 4,8489 \\
\hline Adultos2 & $\begin{array}{c}1,3507^{* * *} \\
(0,0489)\end{array}$ & 3,8600 & $\begin{array}{c}1,0156^{* * *} \\
(0,1623)\end{array}$ & 2,7609 \\
\hline _constante & $\begin{array}{c}-4,6431^{* * *} \\
(0,0428)\end{array}$ & 0,0096 & $\begin{array}{c}-7,2267^{* * *} \\
(0,1332)\end{array}$ & 0,0007 \\
\hline Número de observações & 286.462 & & & \\
\hline Teste F- probabilidade & 0.0000 & & & \\
\hline
\end{tabular}

Nota: $(* * *)=$ significativo a $1 \%$ com base no teste $z_{;}(* *)=$ significativo a $5 \% ;\left({ }^{*}\right)=$ significativo a $10 \%$. Desvio Padrão em parênteses.

Fonte: Elaboração própria com base nos resultados da análise.

As faixas etárias foram estimadas considerando como base de comparação o intervalo de idade Adultos3 (pessoas com mais de 50 anos de idade). O grupo Jovens1 não foi estatisticamente significativo; já Jovens2, Adultos1 e Adultos2 apresentaram chance positiva de estarem em um nível mais alto de rotatividade do que os Adultos3. Os adultos de 36 a 50 anos (Adultos2) foram aqueles com a menor razão de chance de estarem em um nível mais alto de rotatividade.

A comparação entre as pessoas que não saíram do emprego no último ano e as que saíram pelo menos uma vez mostra que os Jovens2 (idade de 18 a 24 anos) foram os que obtiveram maior chance de estarem em um nível mais alto de rotatividade, aproximadamente 7,4 vezes a mais. Estes são seguidos por aqueles com idade entre 25 e 30 
anos de idade, Adultos1, que têm aproximadamente sete vezes a mais de chance do que os Adultos 3 de saírem mais vezes do emprego, no período de 358 dias. Além disso, os Jovens 2 possuem 5,4 vezes mais chances de terem alta rotatividade em comparação com nula e baixa rotatividade.

Os resultados de estimações que incluem importantes variáveis explicativas são apresentados na Tabela 5.

Tabela 5 - Efeitos dos intervalos de idade com as características pessoais, educacionais, familiares, trabalhistas e geográficas sobre os níveis de rotatividade dos trabalhadores brasileiros, 2015

\begin{tabular}{|c|c|c|c|c|}
\hline \multirow{3}{*}{ Variáveis } & \multicolumn{4}{|c|}{ Nível de Rotatividade } \\
\hline & \multicolumn{2}{|c|}{ Nula } & \multicolumn{2}{|c|}{ Baixa } \\
\hline & \multicolumn{2}{|c|}{ Coeficiente Razão de Chance } & \multicolumn{2}{|c|}{ Coeficiente Razão de Chance } \\
\hline \multicolumn{5}{|l|}{ Intervalo de idade } \\
\hline \multirow[t]{2}{*}{ Jovens1 } & $0,9022^{* * *}$ & 2,4652 & $0,9022^{* * *}$ & 2,4651 \\
\hline & $(0,1085)$ & & $(0,1085)$ & \\
\hline \multirow[t]{2}{*}{ Jovens2 } & $1,8783^{* * *}$ & 6,5425 & $1,8783^{* * *}$ & 6,5425 \\
\hline & $(0,0584)$ & & $(0,0584)$ & \\
\hline \multirow[t]{2}{*}{ Adultos1 } & $1,5403^{* * *}$ & 4,6660 & $1,5403^{* * *}$ & 4,6661 \\
\hline & $(0,0501)$ & & $(0,0501)$ & \\
\hline \multirow[t]{2}{*}{ Adultos2 } & $0,8377^{\star * *}$ & 2,3111 & $0,8377^{\star \star \star}$ & 2,3111 \\
\hline & $(0,0515)$ & & $(0,0515)$ & \\
\hline \multicolumn{5}{|l|}{ Características pessoais } \\
\hline \multirow[t]{2}{*}{ Sexo: Masculino } & $0,2275^{\star * *}$ & 1,2555 & $0,2275^{\star * *}$ & 1,2555 \\
\hline & $(0,0265)$ & & $(0,0265)$ & \\
\hline \multirow[t]{2}{*}{ Cor-Raça: Branca-Amarela } & $-0,1618^{* * *}$ & 0,8506 & $-0,1618^{* * *}$ & 0,6783 \\
\hline & $(0,0287)$ & & $(0,0287)$ & \\
\hline \multicolumn{5}{|l|}{ Escolaridade } \\
\hline \multirow[t]{2}{*}{ Anos de escolaridade } & $-0,0352^{* * *}$ & 0,9654 & $-0,1069^{* * *}$ & 0,8986 \\
\hline & $(0,0037)$ & & $(0,0115)$ & \\
\hline \multirow[t]{2}{*}{ Frequenta escola } & $-0,0391$ & 0,9617 & $-0,0391$ & 0,9617 \\
\hline & $(0,0037)$ & & $(0,0037)$ & \\
\hline \multicolumn{5}{|l|}{ Estrutura familiar } \\
\hline \multirow[t]{2}{*}{ Renda dom. per capita } & $-0,00008^{* * *}$ & 0,9999 & $-0,00004^{*}$ & 0,9999 \\
\hline & $(0,00002)$ & & $(0,00002)$ & \\
\hline \multirow[t]{2}{*}{$\mathrm{N}^{\circ}$ de comp. no domicílio } & $-0,0413^{* * *}$ & 0,9595 & $-0,1251^{* * *}$ & 0,8824 \\
\hline & $(0,0088)$ & & $(0,0324)$ & \\
\hline \multirow[t]{2}{*}{ Chefe de família } & $0,2339^{* * *}$ & 1,2635 & $0,2339^{* * *}$ & 1,2635 \\
\hline & $(0,0263)$ & & $(0,0263)$ & \\
\hline \multirow[t]{2}{*}{ Mãe com filhos } & $0,1861^{* *}$ & 1,2045 & $0,1861^{\star * *}$ & 1,2045 \\
\hline & $(0,0854)$ & & $(0,0854)$ & \\
\hline
\end{tabular}




\begin{tabular}{|c|c|c|c|c|}
\hline \multirow{3}{*}{ Variáveis } & \multicolumn{4}{|c|}{ Nível de Rotatividade } \\
\hline & \multicolumn{2}{|c|}{ Nula } & \multicolumn{2}{|c|}{ Baixa } \\
\hline & \multicolumn{2}{|c|}{ Coeficiente Razão de Chance } & \multicolumn{2}{|c|}{ Coeficiente Razão de Chance } \\
\hline \multicolumn{5}{|l|}{ Característica trabalhista } \\
\hline \multirow[t]{2}{*}{ Associado a sindicato } & $-0,0673^{*}$ & 0,9349 & $-0,0673^{*}$ & 0,9349 \\
\hline & $(0,0365)$ & & $(0,0365)$ & \\
\hline \multicolumn{5}{|l|}{ Região Censitária } \\
\hline \multirow[t]{2}{*}{ Urbana } & $0,3115^{* * *}$ & 1,3655 & $0,3115^{\star * *}$ & 1,3655 \\
\hline & $(0,0480)$ & & $(0,0480)$ & \\
\hline \multicolumn{5}{|l|}{ Regiões demográficas } \\
\hline \multirow{2}{*}{ Nordeste } & $0,2578^{\star * *}$ & 1,2941 & $0,5505^{\star * *}$ & 1,7342 \\
\hline & $(0,0263)$ & & $(0,1177)$ & \\
\hline \multirow[t]{2}{*}{ Sul } & $0,6077^{\star * *}$ & 1,8363 & $0,6077^{\star * *}$ & 1,8363 \\
\hline & $(0,0570)$ & & $(0,0571)$ & \\
\hline \multirow[t]{2}{*}{ Sudeste } & $0,4892^{* * *}$ & 1,6309 & $0,4691^{\star * *}$ & 1,6309 \\
\hline & $(0,0501)$ & & $(0,0501)$ & \\
\hline \multirow[t]{2}{*}{ Centro Oeste } & $0,6097^{\star * *}$ & 1,8398 & $0,6096^{\star * *}$ & 1,8398 \\
\hline & $(0,0608)$ & & $(0,0608)$ & \\
\hline \multirow[t]{2}{*}{ _constante } & $-4,2263^{* * *}$ & 0,0146 & $-6,2027^{* * *}$ & 0,0020 \\
\hline & $(0,0867)$ & & $(0,1818)$ & \\
\hline Número de observações & 174.993 & & Stratos & 728 \\
\hline Teste F- probabilidade & 0 & & PSUs & 6332 \\
\hline Teste LR p-valor & 0 & & População & 100.738 .341 \\
\hline Teste R2 MacFadden & 0,044 & & & \\
\hline Count & 0,944 & & & \\
\hline
\end{tabular}

Nota: $(* * *)=$ significativo a $1 \%$ com base no teste $\left.z ;{ }^{* *}\right)=$ significativo a $5 \% ;(*)=$ significativo a $10 \%$. Desvio Padrão em parênteses.

Fonte: Elaboração própria com base nos resultados da análise.

De forma geral, verifica-se que o teste F rejeitou a hipótese de que os coeficientes conjuntamente fossem iguais a zero.

O modelo Logit Ordenado de Razão Proporcionais Parciais é muito parecido com o modelo Logit Ordenado. A diferença está no fato de que somente as variáveis que violaram a hipótese de regressão paralela são relaxadas, por isso, somente algumas das variáveis da Tabela 5 obtiveram resultados diferentes, quando comparados entre os níveis de rotatividade.

De forma similar à especificação anterior, os Jovens2 apresentaram mais chances de terem alta rotatividade, quando comparados aos Adultos3 (mais de 50 anos de 
idade). Nessa mesma linha, Orellano e Picchetti (2001) também observaram, para a região de São Paulo, efeitos decrescentes de rotatividade para trabalhadores no avançar dos anos de idade.

Em relação ao gênero, os homens tiveram maior razão de chances de saírem mais do emprego do que as mulheres, aproximadamente $25 \%$ a mais. Resultado semelhante ao encontrado por Uhr e Uhr (2013), quando consideraram trabalhadores do sexo masculino para o ano de 2009. Ser declarado da cor ou raça branca ou amarela diminuiu em $15 \%$ a chance de estar em um nível mais alto de rotatividade, quando comparados com indivíduos negros e pardos.

Quanto à formação educacional, níveis mais elevados de escolaridade diminuem a probabilidade de uma maior rotatividade no mercado de trabalho (redução de cerca de $4 \%$ na chance de maior alternância, quando se compara nula rotatividade com baixa e alta rotatividade). Interessante ressaltar o contraste disso com o resultado obtido por Monte e Penido (2008), os quais, ao analisarem trabalhadores nordestinos, constataram que indivíduos com maiores níveis de instrução apresentaram rotatividade mais elevada, quando comparados àqueles que possuem somente o ensino fundamental incompleto.

Esses resultados evidenciam a importância da maior escolaridade para a população brasileira, visto que, mantendo-se mais tempo em um emprego, juntamente a maior nível de escolaridade, podem gerar maior produtividade. $\mathrm{O}$ fato de os sujeitos da amostra estarem ou não frequentando a escola na semana de referência não foi estatisticamente significativo.

A análise da estrutura familiar mostra que o número de componentes no domicílio foi significativo na pesquisa realizada. Foi indicado que quanto maior o número de componentes no domicílio, menor a rotatividade dos trabalhadores residentes dessa casa. Isso porque foi observada uma diminuição em $4 \%$ da chance de haver baixa ou alta rotatividade, quando comparado somente com o indivíduo que tem nula rotatividade; e em $12 \%$ de ter alta rotatividade, quando comparado com o indivíduo que tem baixa ou nula rotatividade.

O fato de ser chefe de família aumenta em $26 \%$ a probabilidade de se estar em um nível mais alto de rotatividade. Este resultado não era esperado, pois presumia-se que essa condição de líder familiar tenderia a manter o trabalhador por mais tempo no emprego, como afirmado por Monte e Penido (2008). O panorama atual da economia brasileira pode ser um fator importante para a mudança quanto a esse resultado.

Mães com filhos também apresentaram maiores chances de rotatividade, aproximadamente $20 \%$ a mais do que mulheres sem filhos. A renda domiciliar per capita foi significativa e negativa, indicando que quanto maior a renda domiciliar per capita menor a chance de se estar em nível mais alto de rotatividade. 
Notou-se que pessoas associadas a sindicatos obtiveram menores chances de estarem em nível de maior rotatividade (7\%), quando comparadas aos não associados (esse resultado é estatisticamente significativo a quase 5\%). Esse fato pode ser decorrente de o trabalhador sindicalizado ter maior proteção ao acesso de seus direitos e garantias legais (UHR e UHR, 2013).

Indivíduos que residem em área urbana têm maior probabilidade de saírem mais vezes do emprego, aproximadamente $36 \%$ a mais do que aqueles que residem em área rural. Isso possivelmente acontece devido à maior oferta de trabalho encontrada na área urbana.

As macrorregiões foram comparadas à região Norte. Assim, as regiões Nordeste, Sul, Sudeste e Centro-Oeste obtiveram probabilidades positivas de estarem em um nível mais alto de rotatividade. Dentre esses locais mais suscetíveis à não permanência em um mesmo emprego, as regiões Centro-Oeste e Sul obtiveram as maiores chances de ter alta rotatividade (em torno de $84 \%$ ), seguidas pelas regiões Sudeste (63\%) e Nordeste (29\%), resultados da comparação da nula rotatividade com média e alta rotatividade.

\section{CONSIDERAÇÕES FINAIS}

Entre 2001 e 2015, houve um aumento da população frequente entre os níveis de rotatividade no emprego no Brasil. Além disso, pôde ser observado um percentual maior de nula rotatividade no emprego para o ano de 2015, entre os trabalhadores brasileiros, o que pode, de certo modo, contribuir para o acúmulo de experiência e, consequentemente, de capital humano. Essa maior permanência dos trabalhadores também pode refletir uma resposta dos mesmos em relação à crise política e econômica em que o país se encontra desde o ano de 2014, dada uma menor oferta de empregos e o aumento do risco do desemprego. Dessa forma, este trabalho avaliou os aspectos pessoais que podem determinar as chances individuais de seter maior rotatividade no mercado de trabalho brasileiro.

Em vista disso, os resultados deste artigo evidenciaram que para o ano de 2015 os Jovens 2 e os Adultos1, isto é, de idade de 18 a 35 anos, são os mais propensos a saírem mais do emprego (nível maior de rotatividade) no período de 358 dias, quando comparado aos Adultos3 (mais de 50 anos de idade). Algumas outras características individuais também influenciaram positivamente as chances de maior rotatividade, como o fato de ser do sexo masculino, ser chefe de família, ser mãe com filhos e/ou morar em área urbana.

O resultado de que há elevações nas chances de alta rotatividade quando se é chefe de família ou mãe com filhos não era esperado, visto que trabalhadores com este perfil tendem a possuir maiores preocupações quanto à estabilidade financeira. No entanto, 
esse fato pode refletir certo grau de propensão ao risco, ao maior grau de informalidade dos postos de trabalho desse grupo de indivíduos e à consequente insatisfação no atual emprego.

Quanto à análise do comportamento de jovens e de adultos no mercado de trabalho com relação ao maior nível de rotatividade, evidenciou-se que um importante fator para manter esses trabalhadores de forma mais permanente no mercado de trabalho seria um nível mais elevado escolaridade. Dessa maneira, observa-se a importância da educação que, juntamente à permanência no mercado de trabalho, gera maior produtividade, sendo um resultado essencial para o país que sofre com baixa produtividade em seu mercado de trabalho.

Nesse sentido, torna-se importante salientar que uma possível limitação do trabalho consiste em não saber se a saída do emprego se refere a uma escolha do trabalhador ou do empregador. Diante do atual cenário econômico, essa informação seria de suma importância. Outras variáveis também seriam relevantes no sentido de serem incluídas como preditores do nível de rotatividade no mercado de trabalho, como, por exemplo, as características gerais dos empregos passados (setor que o indivíduo trabalhava antes de sair, salário e formalidade). Diante da base de dados utilizada, essas informações não puderam ser utilizadas, visto que ela não continha maiores informações para trazer maior completude para o trabalho. Assim, para estudos futuros sugere-se a utilização de bancos de dados mais completos quanto ao histórico de emprego dos trabalhadores, como a Relação Anual de Informações Sociais do Ministério do Trabalho (RAIS).

Ademais, este trabalho contribui para a compreensão da dinâmica do mercado de trabalho por apresentar a razão de chance entre diferentes níveis de rotatividade no mercado de trabalho, averiguando as características dos indivíduos que estão mais associadas às probabilidades de apresentarem níveis mais altos de rotatividade. Com o mesmo propósito, este estudo procura trabalhar com dados mais atualizados, podendo, desta forma, captar efeitos do cenário econômico contemporâneo.

\section{REFERÊNCIAS}

ALBUQUERQUE, L. S. G.; PERO, V. Mobilidade de emprego entre os jovens brasileiros. In: ENCONTRO NACIONAL DE ECONOMIA DA ANPEC, 37., Foz do Iguaçu, Paraná, dez. 2009.

ANDRADE, C. C. Juventude e trabalho: alguns aspectos do cenário brasileiro contemporâneo. Mercado de Trabalho: Conjuntura e Análise, IPEA, Rio de Janeiro, n. 37, p. 25-30, 2008.

BARRON, J. M.; McCAFFERTY, S. Job search, labor supply, and the quit decision: theory and evidence. The American Economic Review, v. 67, n. 4, p. 683-691, 1977. 
BARROS, R. P.; CORSEUIL, C. H.; FOGUEL, M. Os incentivos adversos e a focalização dos programas de proteção ao trabalhador no Brasil. Planejamento e Políticas Públicas, n. 22, p. 3-45, 2002.

BECKER, G. Human capital. A theoretical and empirical analysis, with special reference to education. 2. ed. New York: National Bureau of Economic Research, 1975.

BECKER, G.S. Human Capital. 3. ed. Chicago: University of Chicago Press, 1993.

BECKER, G. S. Investment in human capital: A theoretical analysis. Journal of Political Economy, v. 70, n. 5, p. 9-49, 1962.

BLUMEN, I.; KOGAN, M.; MCCARTHY, P. J. The industrial mobility of labor as a probability process. Ithaca, NY: Cornell University Press, 1955.

BURDETT, K. A theory of employee job search and quit rates. The American Economic Review, v. 68, n. 1, p. 212-220, 1978.

CAMARANO, A. A. et al. A transição para a vida adulta: novos ou velhos desafios? Boletim Mercado de Trabalho: Conjuntura e Análise, IPEA, Rio de Janeiro,n. 21, p. 53-66,2003.

CAMERON, A. C.; TRIVEDI, P.K. Microeconometrics - Methods and Applications. New York: Cambridge University Press, 2005.

CORSEUIL, C. H. et al. A rotatividade dos jovens no mercado de trabalho formal brasileiro. Mercado de Trabalho - Conjuntura e Análise, IPEA, Rio de Janeiro, n. 55, 2013.

CORSEUIL, C. H.; RIBEIRO, E. P.; SANTOS, D. Job and Worker Flows in Brazil. Mimeo, 2003.

GONZAGA, G.; MALONEY, W. F.; MIZALA, A. Labor turnover and labor legislation in Brazil. Economía, v. 4, n. 1, p. 165-222, 2003.

IBGE - INSTITUTO BRASILEIRO DE GEOGRAFIA E ESTATÍSTICA. Disponível em: <www. ibge.gov.br>. Acesso em: 11 jun. 2016.

IDRE-UCLA - INSTITUTE FOR DIGITAL RESEARCH AND EDUCATION. Disponível em: $<$ http://www.ats.ucla.edu/stat/stata/dae/ologit.htm>.Acesso em: 11 jun.2016.

JOHNSON, W. R. A theory of Job Shopping. The Quarterly Journal of Economics, v. 92, n. 2, p. 261-277, 1978.

LONG, S. J.; FREESE, J. Regression models for categorical dependent variables using Stata. College Station: Stata Press, 2001.

MENEZES FILHO, N.; PICCHETTI, P. Os determinantes da duração do desemprego em São Paulo. Pesquisa e Planejamento Econômico, Rio de Janeiro, v. 30, n. 1, p. 23-47, 2000.

MINCER, J. Studies in human capital. Collected Essays of Jacob Mincer. v. 1. Cambridge: Edward Elgar, 1993.

MONTE, P. A.; PENILDO, M. R. J. Determinantes da duração esperada do emprego urbano e rural no Nordeste brasileiro. Revista de Economia e Sociologia Rural, São Paulo, v. 46, n. 04, p. $989-1014,2008$.

OI, W. Y. Labor as a quasi-fixed factor of production.unpublished Ph. Dissertation, University of Chicago, Chicago, 1961.

ORELLANO, V., PICCHETTI, P. A bi-variate probit analysis of Job Turnover in Brazil. In: CONGRESSO DA SOCIEDADE BRASILEIRA DE ECONOMETRIA, 23., Salvador, 2001. 
PARSONS, D. O. Specific human capital: An application to quit rates and layoff rates. Journal of Political Economy, v. 80, n. 6, p. 1120-1143, 1972.

RAMOS, C. A.; CARNEIRO, F. G. Os determinantes da rotatividade do trabalho no Brasil: instituições x ciclos econômicos. Nova Economia, Belo Horizonte, v. 12, n. 2, p. 31-55, 2002.

RAMOS, L. O desempenho recente do mercado de trabalho brasileiro: tendências, fatos estilizados e padrões espaciais. Texto para Discussão, IPEA, Rio de Janeiro,n. 1255, 2007.

SINGER, B.; SPILERMAN, S. Some methodologicalissues in theanalysisof longitudinal surveys. In: ANNALS OF ECONOMICS AND SOCIAL MEASUREMENT, NBER, 1976.

SPARROW, P. R. Transitions in the psychological contract: some evidence from the banking sector. Human Resource Management Journal, v. 6, n. 4, p. 75-92, 1996.

UHR, D. A. P.; UHR, J. G. Z. Uma nota sobre a rotatividade da mão-de-obra do setor privado brasileiro. Revista de Economia e Administração, v. 12, n. 1, p. 132-148, 2013.

WILLIAMS, R. Generalized ordered logit/partial proportional odds models for ordinal dependent variables. Stata Journal, v. 6, n. 1, p. 58, 2006.

WILLIAMS, R. Generalized logistic regression/partial proportional odds models for ordinal dependent variables. Disponível em: <http://www.nd.edu/ rwilliam/>. Acesso em: 30 jan. 2017.

WILLIAMS, R. Understanding and interpreting generalized ordered logit models. The Journal of Mathematical Sociology, v. 40, n. 1, p. 7-20, 2016. 